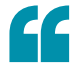

MYC and RAS can induce an immunosuppressive microenvironment
ONCOGENES

\section{Driving immune evasion}

How oncogenes cooperate with each other, and how they direct changes in the tumour microenvironment, especially in immune cells, are intense areas of investigation. Three recent papers have shed light on how the common oncogenic drivers MYC and RAS can induce an immunosuppressive microenvironment.

Kortlever et al. investigated the mechanisms of MYC cooperation with KRAS-G12D in a mouse model of non-small-cell lung cancer (NSCLC). Mice expressing oncogenic $\mathrm{Kras}^{\mathrm{G} 12 \mathrm{D}}$ and inducibly activated $\mathrm{Myc}$ in lung epithelial cells (KM mice) developed aggressive lung adenocarcinomas that were more heavily infiltrated with inflammatory cells and more angiogenic compared with Kras $^{\mathrm{G} 12 \mathrm{D}}$ tumours. Within 24 hours of epithelial $M y c$ activation, stromal changes were observed: macrophage infiltration of tumours increased, and $\mathrm{CD}^{+} \mathrm{T}$ cells, $\mathrm{B}$ cells and natural killer (NK) cells were expelled from tumours. These changes all preceded an increase in tumour size and were promoted by KM tumour cell-derived CC-chemokine ligand 9 (CCL9) and interleukin-23 (IL-23). Depletion of these cytokines reduced tumour development; CCL9 was crucial for infiltration of macrophages, angiogenesis and T cell loss, and IL-23 was crucial for loss of T, B and NK cells. Infiltrating macrophages also expressed programmed cell death 1 ligand 1 (PDL1), which was required for loss of T cells. Myc deactivation in KM mice rapidly reversed the observed stromal changes and induced tumour cell apoptosis and NK cell-dependent regression to the Kras $^{\mathrm{G} 12 \mathrm{D}}$ adenoma state.

Topper et al. also found that MYC drives an immunosuppressive phenotype in NSCLC. They initially investigated

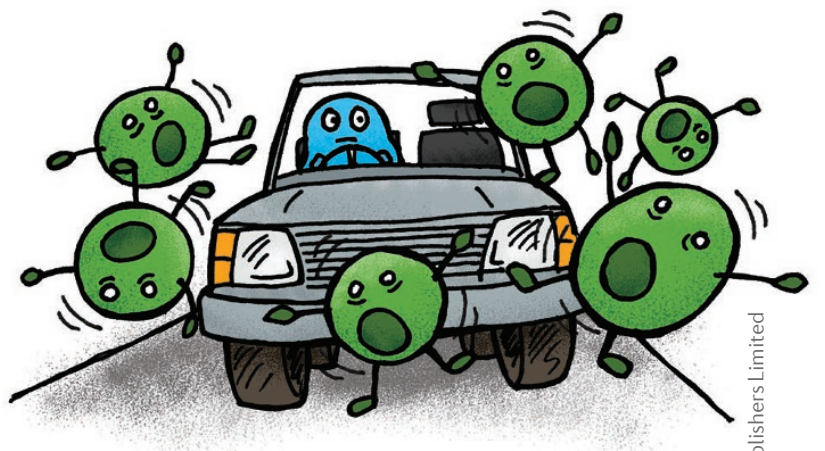
combinations of epigenetic therapies (specifically the DNA methyltransferase inhibitor (DNMTi) azacitidine (aza) with different histone deacetylase inhibitors (HDACis)) and found that aza plus the HDACi ITF-2357 prevented the growth of several RAS-mutant NSCLC cell lines in vitro and in xenograft models. In cells treated with aza plus ITF-2357, the MYC pathway was commonly suppressed and interferon signalling was increased. MYC knockdown phenocopied the aza plus ITF-2357 effect, and MYC overexpression blocked it, suggesting that loss of MYC is important for efficacy. In addition, treatment of two NSCLC mouse models (Kras ${ }^{\mathrm{G} 12 \mathrm{D}}$ and Lewis lung carcinoma (LLC)) with this combination therapy significantly reduced tumour burden, reduced MYC expression and increased interferon signalling. Upon treatment, Kras $^{\mathrm{G} 12 \mathrm{D}}$ mice had decreased numbers of macrophages, reduced angiogenesis and increased numbers of $\mathrm{CD}^{+}$ $\mathrm{T}$ cells, in which exhaustion was reversed. In LLC mice, $T$ cell activation was also observed, and depletion of $\mathrm{CD}^{+} \mathrm{T}$ cells in these mice reduced the efficacy of combination treatment. These authors identified CCL5 as an important factor in $\mathrm{T}$ cell recruitment. Levels of CCL5 were increased in $\mathrm{Kras}^{\mathrm{G} 12 \mathrm{D}}$ mice treated with aza plus ITF-2357, which is consistent with MYC repression of $\mathrm{Ccl} 5$ transcription. These results suggest that the efficacy of immune checkpoint inhibitors might be increased by combined aza and ITF-2357 treatment. In addition, preliminary clinical trial data from four patients have indicated the potential importance of low MYC expression as a biomarker of efficacy of immune checkpoint inhibitors; however, this needs to be confirmed.

Coelho et al. found that oncogenic RAS can also drive immunosuppression. Oncogenic KRAS or HRAS mutants increased the stability of PDL1 mRNA in several cell lines in a manner dependent on downstream MEK activation. The authors identified AU-rich elements (AREs) in the 3' untranslated region (UTR) of PDL1 that negatively regulated its stability. Examination of several ARE-binding proteins indicated that tristetraprolin (TTP) was important for reducing PDL1 mRNA levels, which was controlled by RAS signalling. Several lines of evidence indicated that RASMEK signalling leads to accumulation of reactive oxygen species, which in turn activate p38-MK2 signalling and inactivate TTP through phosphorylation, leading to increased PDL1 stability. Analyses of gene expression data in lung and colon adenocarcinoma indicated that high RAS expression correlated with high PDL1 expression and that TTP is commonly downregulated in these tumours. Finally, subcutaneous growth of mouse colon tumour cells in immunocompetent mice was reduced when TTP expression was induced. This reduction of tumour growth required an intact immune system, specifically $\mathrm{CD}^{+}$ and $\mathrm{CD} 4^{+} \mathrm{T}$ cells.

These papers have uncovered different pathways by which oncogenes impact the immune microenvironment of tumours; understanding this interplay will be important for designing appropriate combination therapies to improve immune eradication of tumours.

Sarah Seton-Rogers

ORIGINAL ARTICLES Kortlever, K. M. et al. Myc cooperates with Ras by programming inflammation and immune suppression. Cell 171, 1301-1315 (2017) | Topper, M. J. et al. Epigenetic therapy ties MYC depletion to reversing immune evasion and treating lung cancer. Cell 171, 1284-1300 (2017) | Coelho, M. A. et al. Oncogenic RAS signaling promotes tumor immunoresistance 1083-1099.e6 (2017) by stabilizing PD-L1 mRNA. Immunity 47, 